\title{
Thermal resistance of the nanoscale constrictions between carbon nanotubes and solid substrates
}

\author{
Hareem Maune, Hsin-Ying Chiu, and Marc Bockrath ${ }^{\mathrm{a})}$ \\ Department of Applied Physics, California Institute of Technology, Pasadena, California 91125
}

(Received 7 March 2006; accepted 17 May 2006; published online 6 July 2006)

\begin{abstract}
We have determined the thermal resistance for transferring heat between individual single-walled carbon nanotube devices and solid substrates. Using sapphire and comparing our results to previous results obtained from $\mathrm{SiO}_{2}$, we find that the resistance is dominated by interfacial resistance rather than the spreading resistance of heat for diffusing into the substrate. Our results are in agreement to a recent model for the thermal resistance of nanoscale constrictions. Our results suggest that relatively short contact lengths $(\sim 10-30 \mathrm{~nm})$ to a typical solid should be sufficient to transfer heat efficiently into carbon nanotubes, underscoring the potential of carbon nanotubes for nanoscale thermal management. (ㅇ 2006 American Institute of Physics. [DOI: 10.1063/1.2219095]
\end{abstract}

As electronic devices are further miniaturized and integration density is increased, the dissipation of heat becomes an important issue for maintaining viability. Thermal management is thus a critical issue towards future nanoelectronic devices. This is important also for obtaining fundamental knowledge about how thermal energy is transported in nanoscale systems. For example, in the nanometer scale, thermal transport differs from the situation in bulk materials because the mean free path for phonon scattering can be large compared to device dimensions, leading to novel physics such as quantum thermal phenomena. ${ }^{1-3}$

Of particular interest are carbon nanotubes, which have been proposed both as prototypical nanoscale circuit elements and by, virtue of their extraordinary current carrying capacity, as potential materials for interconnects. Recent studies have shown that nanotubes are remarkable thermal conductors $^{4-8}$ even at elevated temperatures ${ }^{9,10}$ and ballistic phonon transport has been demonstrated in micron-scale devices, ${ }^{11-13}$ with a mean free path in agreement to theoretical calculations. ${ }^{14}$ Nevertheless, for thermal management it is also of interest to determine the thermal resistance between nanotubes and different materials.

Here we show, using the electrical breakdown phenomenon to perform thermometry, ${ }^{15}$ that in sapphire and $\mathrm{SiO}_{2}$ substrates the dominant thermal resistance between singlewalled nanotubes and solid substrates stems from the substrate-nanotube interface. We find a value of $\sim 3 \mathrm{~K} \mathrm{~m} / \mathrm{W}$ for sapphire substrates compared to $\sim 0.6 \mathrm{~K} \mathrm{~m} \mathrm{~W}^{-1}$ obtained from our previously determined value for multiwalled nanotubes on $\mathrm{SiO}_{2},{ }^{11}$ the value $\sim 12 \mathrm{~K} \mathrm{~m} \mathrm{~W}^{-1}$ obtained from scanned thermal microscopy for multiwalled nanotubes on $\mathrm{SiO}_{2}$ (Ref. 16), and $\sim 2 \mathrm{~K} \mathrm{~m} \mathrm{~W}^{-1}$ based on breakdown data reported for single-walled nanotubes on $\mathrm{SiO}_{2} \cdot{ }^{17}$ Our results are consistent with thermal transport that is limited by the interfacial thermal impedance between the nanotube and substrate, and we find quantitative agreement with a recently reported theoretical model. ${ }^{18}$

Sample fabrication is as follows. Individual singlewalled carbon nanotubes are grown on sapphire by dipping the chip in iron nitrate and then drying after dipping in hexane. The nanotubes are grown under a mixture of $\mathrm{CH}_{4}$ and

\footnotetext{
a)Electronic mail: mwb@caltech.edu
}

$\mathrm{H}_{2}$, and yield predominantly single-walled nanotubes with a fraction of tubes containing multiple shells. ${ }^{19}$ On sapphire substrates, we find aligned nanotube growth on the $a$ and $r$ planes, consistent with the results of Han et al. ${ }^{20}$ Once isolated individual nanotubes are located relative to predefined alignment marks, $\mathrm{Cr} / \mathrm{Au}$ electrodes are placed on the nanotubes using electron beam lithography.

Current voltage measurements are obtained from each tube, and the voltage is increased until the current drops abruptly, signaling electrically driven breakdown. Previous studies indicate that this breakdown occurs when the nanotube reaches a fixed temperature $T_{B}$, which has been estimated to be $\sim 600{ }^{\circ} \mathrm{C} .{ }^{15}$ In addition to thermalized phonons at temperature $T_{B}$, there is also a nonequilibrium population of optical phonons present generated by emission from the electrons. However, these phonons are expected to decay into the thermal population before they can carry significant thermal energy out of the nanotube. ${ }^{11}$

Figure 1 shows $I-V$ curves obtained from individual single-walled carbon nanotube devices for lengths ranging from 0.25 to $8 \mu \mathrm{m}$. The current begins to saturate at larger

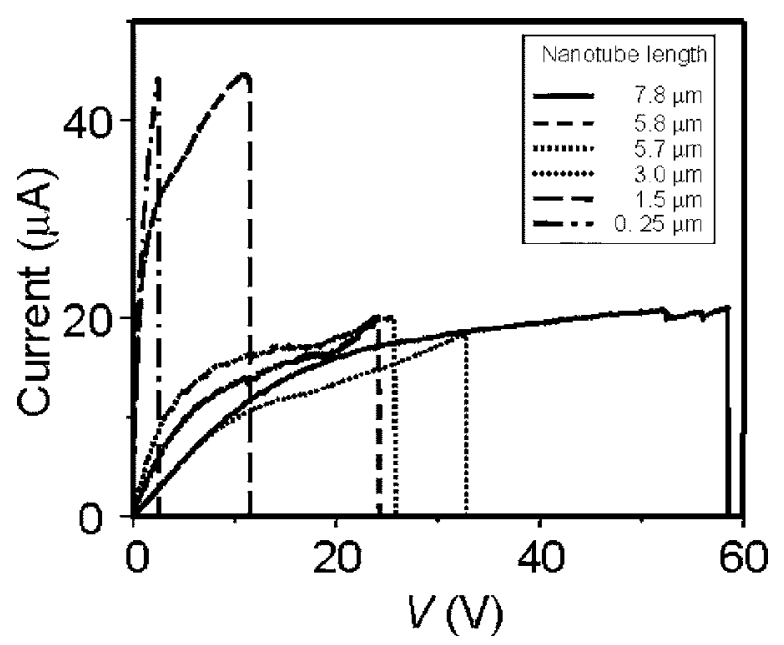

FIG. 1. Current-voltage characteristics for sapphire-supported individual carbon nanotube devices. The voltage is increased until the current drops abruptly due to electrical breakdown. The length of each nanotube is indicated. Longer nanotubes tend to require more electrical power for breakdown. 


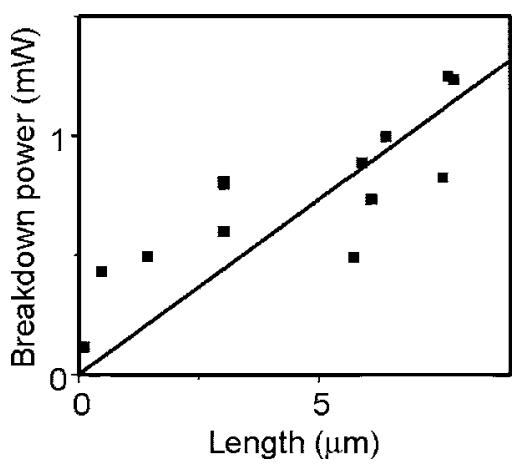

FIG. 2. Breakdown power vs length for sapphire-supported individual carbon nanotube devices. A linear fit to the data is shown with a slope of $0.15 \mathrm{~mW} / \mu \mathrm{m}$.

voltages because of the increased scattering from optical or zone boundary phonon emission, tending towards the maximum of $4 e / h E_{\mathrm{op}-\mathrm{zb}} \approx 25 \mu \mathrm{A} /$ nanotube shell, where $E_{\text {op-zb }} \sim 0.18 \mathrm{eV}$ is the characteristic zone boundary or optical phonon energy in nanotubes. ${ }^{21}$ Although the maximum current can vary for each device, the required power to reach breakdown increases approximately linearly with the nanotube length.

Figure 2 shows a plot of the power required versus length for sapphire substrates. The data follows an approximately linear trend. This indicates that the device cooling occurs along the entire length, and therefore longer nanotubes require more power to reach the same temperature. Fitting a straight line to the data shows that $P=c L$, with $c=0.15 \mathrm{~mW} / \mu \mathrm{m}$. With $T_{B} \sim 600{ }^{\circ} \mathrm{C}$, the thermal resistance on a per-length basis to the substrate can thus be inferred as $\sim 3 \mathrm{~K} \mathrm{~m} / \mathrm{W}$ for sapphire compared to $\sim 0.6-12 \mathrm{~K} \mathrm{~m} / \mathrm{W}$ obtained from previous work for multiwalled nanotubes on $\mathrm{SiO}_{2}$ (Refs. 11 and 16) and $\sim 2 \mathrm{~K} \mathrm{~m} / \mathrm{W}$ single-walled nanotubes on $\mathrm{SiO}_{2}{ }^{17}$ These values are thus similar in magnitude for both $\mathrm{SiO}_{2}$ and sapphire substrates.

However, the thermal conductivity of sapphire is $\sim 40 \mathrm{~W} / \mathrm{K} \mathrm{m}$, while the thermal conductivity of $\mathrm{SiO}_{2}$ is $\sim 1 \mathrm{~W} / \mathrm{K} \mathrm{m}$. We thus expect if cooling were determined by the substrate thermal conductivity that the required breakdown power would be approximately 40 times higher for the case of sapphire as compared to $\mathrm{SiO}_{2}$. Instead, we find similar values. This suggests that the thermal interface resistance, rather than the spreading resistance of thermal energy into the substrate, is the dominant thermal resistance between the nanotube and the substrate. Indeed, in recent work it has been pointed out that consideration of an interfacial boundary thermal resistance is necessary to understand thermal transport across nanoscale boundaries such as the interface between the nanotubes and the substrate. ${ }^{18}$ The author gives the expression

$$
R_{c}=\frac{1}{L}\left[\frac{1}{\pi \kappa_{w}} \ln \left(\frac{2 D}{a}\right)-\frac{1}{2 \kappa_{w}}+\frac{1}{\pi \kappa_{s}} \ln \left(\frac{D}{\pi a}\right)+\frac{R_{b}}{2 a}\right]
$$

for the thermal resistance, where $\kappa_{w}$ is the nanotube thermal conductivity, $\kappa_{s}$ is the substrate thermal conductivity, $D$ is the nanotube diameter, $a$ is the effective contact width, $L$ is the nanotube length, and $R_{b}$ is the interfacial resistance. Since $D$ is much less than the phonon mean free path in nanotubes, the terms with $\kappa_{w}$ can be neglected.

Our data indicate that the term including $R_{b}$ is an important contribution to $R_{c}$. To estimate the order of magnitude of
$R_{b}$, we use the diffuse mismatch (D-M) model ${ }^{18,22,23}$ and apply it to graphite/sapphire or graphite/ $/ \mathrm{SiO}_{2}$ interfaces. In this picture, phonons reaching an interface between two materials are either transmitted across the boundary or reflected with a probability that depends only on the density of phonon modes in each material. This picture is most appropriate for a disordered interface. However, the results obtained from a model based on specular transmission or reflection from the interface typically yield similar results, ${ }^{23}$ and thus we use the D-M model for simplicity. In the D-M model, $R_{b}$ is given by $R_{b}=4 /\left(\alpha_{1 \rightarrow 2} C_{1} v_{1}\right)$, where $C_{1}$ is the specific heat per unit volume, $v_{1}$ is the characteristic speed of sound, and $\alpha_{1 \rightarrow 2}$ is the transmission coefficient for phonons to be transmitted from medium 1 to medium 2. For most solid interfaces $\alpha_{1 \rightarrow 2}$ is a factor of order unity, so for simplicity we take $\alpha_{1 \rightarrow 2}=1$. Using also $v_{1} \sim 5000 \mathrm{~m} / \mathrm{s}$ and $C_{1} \sim 10^{3} \mathrm{~J} / \mathrm{kg} \mathrm{K}$, we find $R_{b} \sim 4 \times 10^{-10} \mathrm{~m}^{2} \mathrm{~K} / \mathrm{W}$. The parameter $a$ can be estimated from the calculated relaxed shape under the van der Waals forces pinning it to the substrates. ${ }^{24}$ For tubes with a characteristic diameter $\sim 2.0 \mathrm{~nm}$ as in our experiment, we expect $2 a \sim 0.3-0.5 \mathrm{~nm}$.

Using the above value for $R_{b}$ and taking $D=2 \mathrm{~nm}$, $\kappa_{\mathrm{SiO}_{2}}=1.0 \mathrm{~W} / \mathrm{m} \mathrm{K}$, and $\kappa_{\text {sapphire }}=40 \mathrm{~W} / \mathrm{m} \mathrm{K}$, we find $R_{c}=1.0 \mathrm{~K} \mathrm{~m} / \mathrm{W}$ (for a unit length) for sapphire and $R_{c} \sim 1.4 \mathrm{~K} \mathrm{~m} / \mathrm{W}$ (for a unit length) for $\mathrm{SiO}_{2}$. These order of magnitude estimates are within a factor of order unity to our measured values of $3 \mathrm{~K} \mathrm{~m} / \mathrm{W}$ for sapphire substrates and previously obtained values by our group and others $\sim 0.6-12 \mathrm{~K} \mathrm{~m} / \mathrm{W}$ for $\mathrm{SiO}_{2}$ substrates. Note also that the values we have obtained are within the typical range observed for the surface thermal resistance between most solids and smaller than the resistance observed between surfactantcoated nanotubes and liquids. ${ }^{25}$ Extrapolating these results suggests that using a contact length $l_{C}$ of only a few tens of nanometers between a typical solid and a nanotube should enable approaching the minimum thermal resistance allowed by quantum mechanics. This minimum value is $\sim 1 / M \kappa_{Q}$, where $M$ is the number of occupied phonon branches, typically 10-20 for a single-walled nanotube at room temperature, and $\kappa_{Q}=\pi^{2} k_{B}{ }^{2} T / 3 h$ is the thermal conductance quantum. ${ }^{1,2}$ Note that this assumes ballistic phonon transport. For sufficiently long nanotubes, phonon transport is diffusive and the nanotube thermal resistance would be larger than the quantum mechanical limit above. The above estimate for $l_{C}$ is thus an upper bound on the required contact length so that the intrinsic nanotube thermal resistance is dominant. Nevertheless, this prediction should be supported by more accurate calculations, such as molecular dynamics simulations and further experiments.

In sum, we have determined the thermal resistance between carbon nanotubes and sapphire substrates using nanotube electrical breakdown to determine the temperature. We find that the thermal resistance is dominated by the interfacial thermal resistance and is little affected by the substrate thermal conductivity for both $\mathrm{SiO}_{2}$ and sapphire. Our measured values for the interfacial resistance between nanotubes and sapphire agree with recent theory using order of magnitude estimates for the surface resistance based on the diffuse mismatch model. Our results suggest that relatively short contact lengths $(\sim 10 \mathrm{~nm})$ to a typical solid can transfer heat efficiently into nanotubes, further underscoring their potential for thermal management in nanoscale circuits.

o AIP license or copyright, see http://apl.aip.org/apl/copyright.jsp 
This work was supported by the ONR, the Powel Foundation, and the NSF. The authors thank Natalio Mingo for helpful discussions.

${ }^{1}$ K. Schwab, E. A. Henriksen, J. M. Worlock, and M. L. Roukes, Nature (London) 404, 974 (2000).

${ }^{2}$ J. B. Pendry, J. Phys. A 16, 2161 (1983).

${ }^{3}$ R. Maynard and E. Akkermans, Phys. Rev. B 32, 5440 (1985).

${ }^{4}$ J. Hone, M. Whitney, and A. Zettl, Synth. Met. 103, 2498 (1999).

${ }^{5}$ J. Hone, B. Batlogg, Z. Benes, A. T. Johnson, and J. E. Fischer, Science 289, 1730 (2000).

${ }^{6}$ M. C. Llaguno, J. E. Fischer, A. T. Johnson, and J. Hone, Nano Lett. 4, 45 (2004).

${ }^{7}$ J. P. Small, K. M. Perez, and P. Kim, Phys. Rev. Lett. 91, 256801 (2003).

${ }^{8}$ P. Kim, L. Shi, A. Majumdar, and P. L. McEuen, Phys. Rev. Lett. 87, 215502 (2001).

${ }^{9}$ E. Pop, D. Mann, J. Cao, Q. Wang, K. Goodson, and H. J. Dai, Phys. Rev. Lett. 95, 155505 (2005).

${ }^{10}$ E. Pop, D. Mann, Q. Wang, K. Goodson, and H. J. Dai, Nano Lett. 6, 96 (2006).

${ }^{11}$ H. Y. Chiu, V. V. Deshpande, H. W. C. Postma, C. N. Lau, C. Miko, L. Forro, and M. Bockrath, Phys. Rev. Lett. 95, 226101 (2005).
${ }^{12}$ C. H. Yu, L. Shi, Z. Yao, D. Y. Li, and A. Majumdar, Nano Lett. 5, 1842 (2005).

${ }^{13}$ E. Brown, L. Hao, J. C. Gallop, and J. C. Macfarlane, Appl. Phys. Lett. 87, 023107 (2005).

${ }^{14}$ N. Mingo and D. A. Broido, Phys. Rev. Lett. 95, 096105 (2005).

${ }^{15}$ P. G. Collins, M. Hersam, M. Arnold, R. Martel, and P. Avouris, Phys. Rev. Lett. 86, 3128 (2001).

${ }^{16}$ P. Kim, L. Shi, A. Majumdar, and P. L. McEuen, Physica B 323, 67 (2002).

${ }^{17}$ A. Javey, J. Guo, M. Paulsson, Q. Wang, D. Mann, M. Lundstrom, and H. J. Dai, Phys. Rev. Lett. 92, 106804 (2004).

${ }^{18}$ R. Prasher, Nano Lett. 5, 2155 (2005).

${ }^{19}$ J. Kong, H. T. Soh, A. M. Cassell, C. F. Quate, and H. Dai, Nature (London) 395, 878 (1998).

${ }^{20}$ S. Han, X. L. Liu, and C. W. Zhou, J. Am. Chem. Soc. 127, 5294 (2005).

${ }^{21}$ Z. Yao, C. Kane, and C. Dekker, Phys. Rev. Lett. 84, 2941 (2000).

${ }^{22}$ E. T. Swartz and R. O. Pohl, Rev. Mod. Phys. 61, 605 (1989).

${ }^{23}$ D. G. Cahill, W. K. Ford, K. E. Goodson, G. D. Mahan, A. Majumdar, H. J. Maris, R. Merlin, and S. R. Phillpot, J. Appl. Phys. 93, 793 (2003).

${ }^{24}$ T. Hertel, R. E. Walkup, and P. Avouris, Phys. Rev. B 58, 13870 (1998).

${ }^{25}$ S. T. Huxtable, D. G. Cahill, S. Shenogin, L. P. Xue, R. Ozisik, P. Barone, M. Usrey, M. S. Strano, G. Siddons, M. Shim, and P. Keblinski, Nat. Mater. 2, 731 (2003). 